2014 to Sep 2019. Patients complicated with pulmonary artery hypertension deep venous thrombosis or pulmonary embolism and underwent cardiac surgery during the follow-up period were excluded. Left ventricular(LV) systolic dysfunction was defined as ejection fraction $(E F)<50 \%$. LV diastolic dysfunction was defined according to ASE/EACVI guideline ${ }^{4}$. LV dysfunction (LVD) includes one or both of LV systolic dysfunction and LV diastolic function. Monocyte to HDL ratio (MHR) was calculated by dividing monocyte count with HDL-C level.

Prevalence of left ventricular abnormalities was analysed at baseline and follow-up examination. Clinical characteristics and laboratory data were compared among patient groups as follows; patients with LV dysfunction (Group A) and without LV dysfunction (Group B) at the follow-up echocardiography, patients with LV asynergy at any point of examination (Group C) and patients free of LV abnormalities during the follow-up period (Group D).

Results: At the baseline examination, LV dysfunction (5/29 cases, $13.8 \%$ ), LV asynergy $(6 / 29$ cases, $21.7 \%)$ were detected. Pericarditis was detected in 7 patients (24.1\%, LVD in 3 patients, LV asynergy in 2 patients) and 2 of them with subacute onset had progressive LV dysfunction, while 5 patients were normal in echocardiography after remission induction therapy for SLE. At the follow-up examination, LV dysfunction (9/29 cases, 31.0\%, 5 new-onset and 1 improved case), LV asynergy (6/29 cases, $21.7 \%, 2$ new-onset and 2 improved cases) were detected. Though any significant differences were observed between Group A and Group B at the baseline, platelet count (156.0 vs 207.0, $p=0.049$ ) were significantly lower in LV dysfunction group (Group A) at the follow-up examination. Group $C$ patients had significantly higher uric acid $(p=0.004)$, monocyte count $(p=0.009)$, and MHR $(p=0.003)$ than Group $D$ (results in table).

Conclusion: LV dysfunction is progressive in most of patients and requires regular follow-up once they developed. Uric acid, monocyte count and MHR are elevated in SLE patients with LV asynergy. Since MHR elevation was reported as useful marker of endothelial dysfunction ${ }^{5}$, our future goal is to analyse involvement of monocyte activation and endothelial dysfunction in LV asynergy of SLE patients.

References:

[1] Doria A et al. Lupus. 2005;14(9):683-6.

[2] Manger K et al. Ann Rheum Dis. 2002 Dec;61(12):1065-70.

[3] Leone P et al. Clin Exp Med. 2019 Dec 17.

[4] Nagueh SF et al. J Am Soc Echocardiogr. 2016 Apr;29(4):277-314.

[5] Acikgoz N et al. Angiology. 2018 Jan;69(1):65-70.

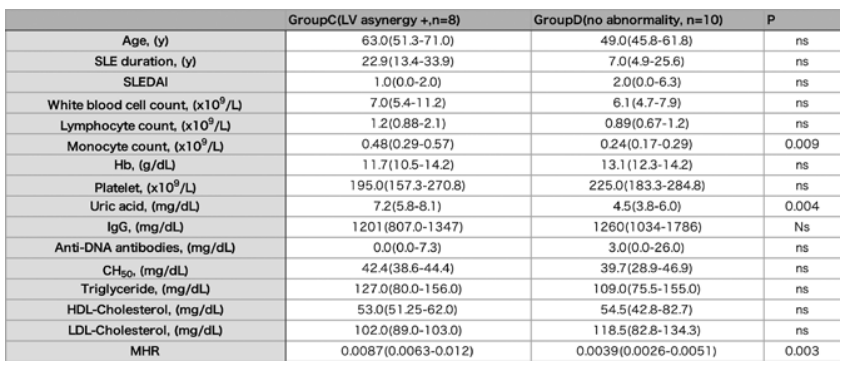

Numbers are median (interquartile range), Mann-Whitney u test were performed, $p$ value less than 0.05 was considered statistically significant.

Disclosure of Interests: : None declared

DOI: 10.1136/annrheumdis-2020-eular.1941

\section{AB0423 NEUROPSYCHIATRIC OUTCOME OF CHILDREN BORN TO WOMEN WITH SYSTEMIC LUPUS ERYTHEMATOSUS (SLE) WOMEN AND EXPOSED IN UTERO TO AZATHIOPRINE: A CASE-CONTROL STUDY.}

M. G. Lazzaroni ${ }^{1}$, F. Crisafulli ${ }^{1}$, I. Debeni ${ }^{1}$, C. Nalli ${ }^{1}$, L. Andreoli ${ }^{1}$, J. Galli ${ }^{1}$, A. Merlini ${ }^{1}$, E. Fazzi ${ }^{1}$, F. Franceschini ${ }^{1}$, A. Tincani ${ }^{1} .{ }^{1}$ ASST Spedali Civili of Brescia, University of Brescia, Brescia, Italy

Background: A possible increase in neurodevelopmental (ND) and learning disorders (LD) in the offspring of mothers affected by SLE have been suggested in some studies, along with the identification of different possible risk factors. Azathioprine (AZA) is commonly used during pregnancy, based on its non-teratogenicity and extended experience in women with different diseases. However, a few small studies suggested an association between in utero exposure to AZA and possible increased frequency of ND/LD in children, indirectly derived from increased request of supportive educational services.

Objectives: To evaluate the medium-long term outcome in terms of ND/LD in children of school age ( $\geq 6$ years) born to SLE women treated with AZA during pregnancy, as compared to that of children born to SLE mothers not treated with AZA during pregnancy.
Methods: Data from our Pregnancy Clinic registry were collected for prospectively followed pregnancies of SLE women treated with AZA (cases) and compared to pregnancies of SLE women not treated with AZA (controls), that were matched for age at pregnancy, presence of renal involvement and aPL positivity. SLE patients (cases and controls) were interviewed by phone to collect data about their children focusing on the presence of ND/LD certified by Neuropsychiatrists.

Results: Data were collected for 14 SLE mothers in the AZA group and 31 in the control group, with similar age at pregnancy $(30.3 \pm 5.21$ vs $31.4 \pm 4.70$ years, $\mathrm{p}: 0.45)$ and frequency of renal involvement (50.0\% vs $44.1 \%, \mathrm{p}: 0.77)$, aPL positivity (33.3\% vs $29.4 \%$, p:0.76) and anti-Ro/SSA positivity ( $27.8 \%$ vs. $26.5 \%$ p:0.55). A SLE flare during pregnancy was more frequently recorded in the AZA group (27.8\% vs. $2.94 \%$, p:0.02). Other medications included HCQ $(55.6 \%$ vs $70.6 \%, \mathrm{p}: 0.36)$ and corticosteroids (100\% vs $79.4 \%, \mathrm{p}: 0.08)$.

We collected data for 18 children in the AZA group and 34 children in the control group, that had a similar mean age at the time of the interview $(12.7 \pm 4.80$ vs. $12.9 \pm 5.61$ years, p:0.91). The two groups had also similar gestational age $(37.4 \pm 2.20$ weeks vs. $38.0 \pm 1.29$ weeks, p:0.23), birth weight $(3003 \pm 433 \mathrm{~g}$ vs $3011 \pm 453 \mathrm{~g}, \mathrm{p}: 0.95)$ and rate of male sex (61.1\% vs $44.1 \%, \mathrm{p}: 0.38)$.

We recorded similar frequency of ND/LD in the two groups. In particular, a ND was present in $2 / 18(11.1 \%)$ of children exposed to AZA vs. $2 / 34(5.88 \%)$ in the control group (p:0.60). A LD was present in $1 / 18$ cases $(5.56 \%)$ and $6 / 34$ controls (17.6\%) (p:0.40).

Conclusion: The medium-long term outcome of children born to SLE mothers in the whole cohort was characterized by the presence of ND in $4 / 54(7.69 \%)$ and LD in $7 / 52$ (13.5\%). ND/LD do not seem to be related to in utero exposure to AZA Disclosure of Interests: None declared

DOI: 10.1136/annrheumdis-2020-eular.4912

\begin{tabular}{l|l}
\hline AB0424 & PREDICTORS OF SLE FLARE-UP AND PREMATURE \\
DELIVERY IN PREGNANCY
\end{tabular}

C. H. Liao ${ }^{1}$, L. C. Wang ${ }^{2}$, S. C. Hsieh ${ }^{3}$, B. L. Chiang ${ }^{2} .{ }^{1}$ National Taiwan University Hospital, Pediatric, Taipei, Taiwan, Republic of China; ${ }^{2}$ National Taiwan University Hospital, Pediatric, Taipei, Taiwan, Republic of China; ${ }^{3}$ National Taiwan University Hospital, Internal medicine, Taipei, Taiwan, Republic of China

Background: Systemic lupus erythematosus (SLE) is a life-threatening autoimmune disease that affects many women of child-bearing age, with potentially severe consequences on pregnancy outcome. SLE flare-ups may occur during pregnancy and the post-partum period. Previous studies documenting the incidence and risk factors of flare-up of SLE during pregnancy and puerperium had partially discordant results.

Objectives: We aimed to delineate the pregnancy complications of women with SLE, as well as neonatal outcomes of their offspring, and hoped to clarify the incidence and risk factors of SLE flare-ups during pregnancy and puerperium.

Methods: We retrospectively reviewed the medical records of SLE patients with previous records of pregnancies in our institution. Flare events during pregnancy and puerperium were documented. The pregnancy outcomes recorded include live births, intra-uterine fetal death (IUFD), premature delivery $(<36$ weeks of gestational age), NICU admission, and small for gestational age (SGA, $<10^{\text {th }}$ percentile). Univariate logistic regression was performed to determine the factors associated with disease relapse and pregnancy outcomes.

Results: From January, 2000 to December, 2019, a total of 94 SLE patients with 139 pregnancies were identified. The overall live birth rate was $92.4 \%$ (134/145). Forty-six (34.3\%) of the neonates were delivered prematurely. Forty-six (34.3\%) of them were SGA. The admission rate to the neonatal intensive care unit was $25 \%(30 / 120)$. Nine (6.4\%) were diagnosed to have SLE during pregnancy. The flare rate during pregnancy was $20 \%$ while post-partum $9.4 \%$. The majority of the relapses during pregnancy occurred in the second trimester $(46.2 \%)$, followed by the first trimester $(30.8 \%)$, and the third trimester $(23.1 \%)$. Low complement C3 $(\mathrm{C} 3<80 \mathrm{mg} / \mathrm{dl})$, thrombocytopenia $\left(\mathrm{PLT}<100^{\star} 10^{3} / \mathrm{uL}\right)$ at conception, and low serum albumin level at the first trimester were associated with antepartum flare. Presence of disease flare and pre-eclampsia in pregnancy, and low serum albumin level at conception were significantly associated with premature delivery. Conclusion: Low complement C3 and thrombocytopenia at conception, and low serum albumin level at the first trimester were associated with disease flare-up during pregnancy. Patients with relative low serum albumin level at conception, or presence of eclampsia or disease flare-up during pregnancy had a higher risk of premature delivery.

References:

[1] Shaharir SS, Mohamed Said MS, Mohd R, et al. Predictors of SLE relapse in pregnancy and post-partum among multi-ethnic patients in Malaysia. PloS one 2019;14(9):e0222343.

[2] Bundhun PK, Soogund MZ, Huang F. Impact of systemic lupus erythematosus on maternal and fetal outcomes following pregnancy: A meta-analysis of studies published between years 2001-2016. Journal of autoimmunity 2017;79:17-27. 
Disclosure of Interests: : None declared DOI: 10.1136/annrheumdis-2020-eular.2904

\section{AB0425 PRELIMINARY EVALUATION OF THE SURAL NERVE USING 24-MHZ ULTRASOUND: A NEW APPROACH TO EVALUATE SURAL NEUROPATHY OF SJOGREN'S SYNDROME PATIENTS}

F. Liu ${ }^{1}$, J. Zhu', S. Zhang ${ }^{1}$, D. LI ${ }^{1}$, F. Liu', W. LI ${ }^{1}$, Y. Liu ${ }^{1} .{ }^{1}$ Peking University People's Hospital, Department of Ultrasound, Beijing, China

Background: Peripheral neuropathy is one of the most frequent extraglandular manifestations of primary Sjögren's syndrome (pSS). The diagnosis of peripheral neuropathy complications of pSS is based primarily on careful neurologic examination and electrodiagnostic tests. The value of ultrasound in peripheral nerve has been recognized. However, little clinical researches have focused specifically on cutaneous nerve of pSS.

Objectives: To evaluate the morphological changes of sural nerve in patients with $\mathrm{pSS}$ by high-frequency ultrasound.

Methods: The prospective study subjects consisted of 31 consecutive pSS patients underwent sural nerve biopsy and 30 healthy volunteers as controls. The ultrasonic presentations of the fascicle, perineurium, epineurium of sural nerve were observed, and the cross-sectional areas (CSA) of the sural nerves was measured.

Results: Among the 21 sural nerves confirmed by pathology, all showed the thickening of the perineurium and epineurium (Figure 1-2), and abnormal blood flow signal in perineurium or epineurium in 14 cases (Figure 2). The mean CSAs were $(1.41 \pm 0.44) \mathrm{mm} 2$ for the control group, and $(1.58 \pm 0.48) \mathrm{mm} 2$ for the case group $(P>0.05)$. In addition, the abnormal blood flow signal in sural nerve correlated with disease activity.

Conclusion: This study indicated that high-frequency ultrasound may be a valuable tool for evaluating cutaneous nerve neuropathy of Sjogren's syndrome patients.

References:

[1] Vitali C, Bombardieri S, Jonsson R, Moutsopoulos HM, Alexander EL, Carsons SE, et al. Classification criteria for Sjögren's syndrome: a revised version of the European criteria proposed by the American-European Consensus Group. Ann Rheum Dis. 2002;61(6):554-8.

[2] Terrier B, Lacroix C, Guillevin L, Hatron PY, Dhote R, Maillot F, et al. Diagnostic and prognostic relevance of neuromuscular biopsy in primary Sjögren's syndrome-related neuropathy. Arthritis Rheum.2007;57(8):1520-9.

[3] McCoy SS, Baer AN. Neurological Complications of Sjögren's Syndrome: Diagnosis and Management. Curr Treatm Opt Rheumatol. 2017;3(4):275-88.

[4] Carvajal Alegria G, Guellec D, Devauchelle-Pensec V, Saraux A. Is there specific neurological disorders of primary Sjögren's syndrome? Joint Bone Spine. 2015;82(2):86-9.

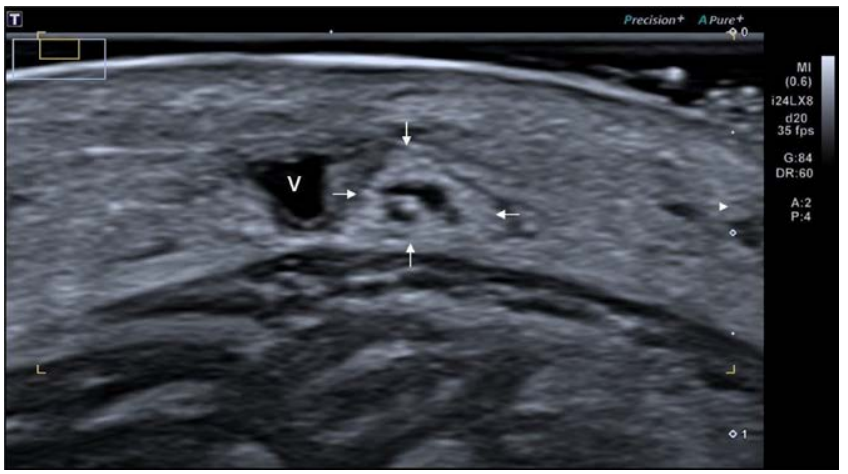

Figure 1. Transverse sonograms of the sural nerve (arrows)V: indicates lesser saphenous vein

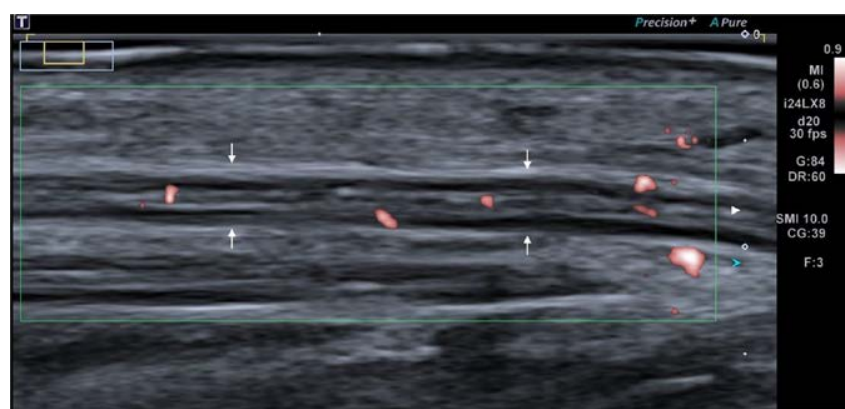

Figure 2. Longitudinal sonograms of the sural nerve (arrows)The sonogram of sural nerve showed abnormal blood flow signal.V indicates lesser saphenous vein.

Acknowledgments: This work was partly supported by National Natural Science Foundation of China (No. 81701712)

Disclosure of Interests: : None declared

DOI: 10.1136/annrheumdis-2020-eular.4176

\section{AB0426 1 FIBROSING INTERSTITIAL LUNG DISEASE IN PRIMARY SJOGREN SYNDROME}

A. Manfredi ${ }^{1}$, C. Vacchi ${ }^{1}$, G. Della Casa ${ }^{1}$, S. Cerri ${ }^{1}$, G. DI Cecco ${ }^{1}$, F. Coppi ${ }^{1}$, F. Luppi ${ }^{2}$, C. Salvarani ${ }^{1}$, M. Sebastiani ${ }^{1}{ }^{1}$ University of Modena and Reggio Emilia, Modena, Italy; ${ }^{2}$ University of Milano-Bicocca, Monza, Italy

Background: Interstitial lung disease (ILD) represents the most frequent pulmonary manifestation of primary Sjogren's syndrome (pSS), with a prevalence ranging between $6-70 \%$ in different retrospectives studies. Non-specific interstitial pneumonia (NSIP) is recognized as the most common ILD disorder, followed by organizing pneumonia (OP), usual interstitial pneumonia (UIP) and lymphocytic interstitial pneumonia (LIP), specifically associated with pSS but less frequent. Objectives: To investigate the prevalence of fibrosing patterns in a monocentric cohort of pSS patients evaluated for lung involvement in a cross-sectional study. Methods: In a cross-sectional study all patients fulfilling ACR/EULAR classification criteria for pSS and with a known diagnosis of ILD were enrolled; the other patients were carefully investigated for signs or symptoms suggestive for ILD (including the search for velcro crackles with a digital device); when suspect, patients underwent to high resolution computed tomography (HRCT). An expert radiologist re-evaluated all HRCT for classifying the ILD pattern as: UIP, fibrotic NSIP, fibrotic OP, NSIP, OP, LIP, indeterminate. Results: One hundred and eighty-five pSS patients were enrolled; among them 34 showed ILD (18.4\%) with the following features: M/F 3/31, median age 57 (range 24-80), median FVC 90\% (39-127\%), median DLCO 49\% (20-84\%). Patients were classified in two groups according to radiologic classification: the group 1 (18 pts 52,9\%) included UIP (13 patients, 38.2\%), fibrotic NSIP (4, 11.8\%), fibrotic OP (12.9\%); the group 2 (16 pts, $47.1 \%)$ included NSIP $(6,17.6 \%)$, OP $(4,11.8 \%)$, indeterminate $(4,11.8 \%)$, LIP (2 $5.9 \%)$. No significant differences were observed between the two groups with the exception of anti-SSB positivity more frequently detected in non-fibrosing pattern ( $p 0,043)$. Conclusion: Despite previous observations, our data suggest a high prevalence of fibrosing ILD pattern in pSS patients. We participate at a multidisciplinary team with expert pulmonologists and radiologists and some patients of our cohort firstly referred to pulmonologist for appearance of ILD before the diagnosis of pSS, contributing to the possible selection of more severe lung disease. However, these data suggest first of all that pSS should always be considered in differential diagnosis of fibrosing ILD; moreover, since fibrosing ILD is thought to have a worse response to immunosuppressive drugs, the role of new possible therapeutic strategies such as anti-fibrotic could represent an important field of interest.

Disclosure of Interests: None declared

DOI: 10.1136/annrheumdis-2020-eular.5769

\section{AB0427 OCCURRENCE OF NEUROPSYCHIATRIC MANIFESTATIONS IN PATIENTS WITH SYSTEMIC LUPUS ERYTHEMATOSUS: RESULTS FROM THE SWISS LUPUS COHORT AND META-ANALYSIS OF THE PERTINENT LITERATURE}

A. L. Meier ${ }^{1,2}$, U. Steiner ${ }^{1}$, L. M. Bachmann ${ }^{2} .{ }^{1}$ University Hospital of Zürich, Dept. of Immunology, Zürich, Switzerland; ${ }^{2}$ Medignition Healthcare Innovations, Zürich, Switzerland 